\title{
Deciphering the 'fuzzy' interaction of FG nucleoporins and transport factors using
}

\section{SANS}

David Cowburn*, Samuel Sparks ${ }^{1}$, Deniz B. Temel $^{1,3}$, Michael P. Rout ${ }^{2}$

${ }^{1}$ Departments of Biochemistry and of Physiology \& Biophysics, Albert Einstein College of Medicine, Bronx, NY, USA

2

Laboratory of Cellular and Structural Biology, Rockefeller University, New York, NY, USA

${ }^{3}$ Current address - Amgen Inc., Thousand Oaks, CA, USA

*Correspondence: david.cowburn@einstein.yu.edu

ABSTRACT

The largely intrinsically disordered phenylalanine-glycine-rich nucleoporins (FG Nups) underline a selectivity mechanism which enables the rapid translocation of transport factors (TFs) through the nuclear pore complexes (NPCs). Conflicting models of NPC transport have assumed that FG Nups undergo different conformational transitions upon interacting with TFs. To selectively characterize conformation changes in FG Nups induced by TFs we performed small-angle neutron scattering (SANS) with contrast matching. Conformational ensembles derived SANS data indicate an increase in the overall size of FG Nups is associated with transport factor interaction. Moreover, the organization of the FG motif in the interacting state is consistent with prior experimental analyses defining that FG motifs undergo conformational restriction upon interacting with TFs. These results provide structural insights into a highly dynamic interaction and illustrate how functional disorder imparts rapid and selective FG Nup - TF interactions. 